\title{
Range imager performance comparison in homodyne and heterodyne operating modes
}

\author{
Richard M. Conroy ${ }^{*}$, Adrian A. Dorrington, Rainer Künnemeyer, and Michael J. Cree. \\ Department of Engineering, University of Waikato, Private Bag 3105, Hamilton, New Zealand.
}

\begin{abstract}
Range imaging cameras measure depth simultaneously for every pixel in a given field of view. In most implementations the basic operating principles are the same. A scene is illuminated with an intensity modulated light source and the reflected signal is sampled using a gain-modulated imager. Previously we presented a unique heterodyne range imaging system that employed a bulky and power hungry image intensifier as the high speed gain-modulation mechanism. In this paper we present a new range imager using an internally modulated image sensor that is designed to operate in heterodyne mode, but can also operate in homodyne mode. We discuss homodyne and heterodyne range imaging, and the merits of the various types of hardware used to implement these systems. Following this we describe in detail the hardware and firmware components of our new ranger. We experimentally compare the two operating modes and demonstrate that heterodyne operation is less sensitive to some of the limitations suffered in homodyne mode, resulting in better linearity and ranging precision characteristics. We conclude by showing various qualitative examples that demonstrate the system's three-dimensional measurement performance.
\end{abstract}

Keywords: Three-dimensional, range, camera, imaging, homodyne, heterodyne, PMD, gain modulation

\section{INTRODUCTION}

Range imaging systems based on indirect time-of-flight measurement techniques determine both distance and intensity simultaneously for every pixel in an image. These systems are capable of effectively measuring the size, shape and location of objects in a scene thereby enabling many new applications in areas such as automotive safety and control, multimedia user interface, image segmentation and surface profiling. The parallel nature of the range acquisition means depth images can be acquired at video frame rates, yet systems can have the flexibility to operate in slower modes to achieve sub-millimeter depth resolution ${ }^{1}$.

Numerous systems based on common fundamental operating principles are being developed by various groups. Typically a scene is illuminated with an intensity-modulated light source and the reflected signal is sampled using a gainmodulated imager. The frequencies used to drive the gain modulation (also called the high-speed shutter) and light source are usually between $10 \mathrm{MHz}$ and $100 \mathrm{MHz}$. Distance is determined by calculating the phase change in the modulation envelope of the returned illumination for each pixel.

The most common approach is to use a homodyne (with quadrature) detection scheme, which is known to suffer a number of limitations such as unwanted harmonics in the output signal ${ }^{2}$. Previously we presented a range imaging camera that operates in an alternative heterodyne mode $e^{1,3,4}$. This system utilized an image intensifier to perform the high speed shuttering in front of a standard Charge Coupled Device (CCD) camera. But although this configuration can deliver excellent depth uncertainty at relatively high spatial resolutions (512-by-512 pixels), the excessive power requirements and large size of the intensifier limit the system's application potential.

An alternative technology is internally modulated custom image sensors where the high-speed shutter is integrated onchip as part of the pixel architecture. We have developed an alternative heterodyne range imaging system using one of these devices (developed by PMD Technologies $\mathrm{GmbH}$, described in section 4 below). Built primarily for implementing and characterizing our heterodyne configuration with this type of hardware, the system also has the flexibility to operate in the homodyne mode allowing us to compare the measurement performance of both configurations.

*rmc21@waikato.ac.nz

Three-Dimensional Imaging Metrology, edited by J. Angelo Beraldin, Geraldine S. Cheok, Michael McCarthy,

Ulrich Neuschaefer-Rube, Proc. of SPIE-IS\&T Electronic Imaging, SPIE Vol. 7239, 723905

(c) 2008 SPIE-IS\&T · CCC code: 0277-786X/08/\$18 - doi: 10.1117/12.806139 
In this paper we will describe this new ranger in detail including a description of its various hardware and firmware components. Also included are explanations of both heterodyne and homodyne modes of operation as well as a discussion of the two fundamental types of imaging hardware that are used for these systems (internal versus external image modulation). Using various experiments we will broadly examine and compare the system's measurement performance in both heterodyne and homodyne operating modes. Finally we will include qualitative measurement examples to demonstrate the camera's three-dimensional measurement capabilities.

\section{HOMODYNE AND HETERODYNE RANGE IMAGING}

Time-of-flight range imaging systems determine the small amount of time light takes to travel to an object and back (and therefore distance to the object) for every pixel in an image. A common and practical approach to is to illuminate the scene of interest using an intensity-modulated light source and sample the backscattered signal using a gain modulated (or shuttered) imager. The round-trip propagation delay causes a phase shift in the received illumination modulation envelope. Given this phase shift $(\varphi)$, distance to the object $(d)$ can be determined using equation (1) where $c$ is the speed of light and $f$ is the modulation frequency, which for these systems is typically around 10-100 MHz.

$$
d=\frac{\varphi c}{4 \pi f}
$$

Typically a homodyne configuration is employed where the modulation signals applied to the light source and sensor are identical. In this mode the phase-shifted illumination is mixed with the camera shutter to produce a constant brightness level $^{5}$. Although distance can be deduced from this value, variations in factors such as object color, texture and background lighting cause far too much interference rendering the results unusable.

To overcome these limitations a quadrature detection scheme is used where four images are acquired with the relative phase of the two modulation signals advanced by 90 degrees for each sample ${ }^{6}$. From the four values recorded for each pixel ( $A_{0}, A_{90}, A_{180}$ and $\left.A_{270}\right)$ the phase, $\varphi$, and signal amplitude, $\alpha$, can be calculated using equations (2) and (3).

$$
\begin{gathered}
\varphi=\tan ^{-1}\left(\frac{A_{0}-A_{180}}{A_{90}-A_{270}}\right) \\
\alpha=\frac{\sqrt{\left(A_{0}-A_{180}\right)^{2}+\left(A_{90}-A_{270}\right)^{2}}}{2}
\end{gathered}
$$

An alternative configuration, called heterodyne, uses slightly different frequencies for the two modulation signals. In this mode the mixing effect between the reflected illumination and camera shutter produces a beat signal at the detector with a frequency equal to the difference between the two signal frequencies (typically singles to tens of Hertz) ${ }^{3}$. The phase delay of the illumination waveform is preserved in the low frequency beat and can be determined through analysis of the time varying intensities for each pixel. This concept can be observed in a captured video sequence where objects in a scene flash on and off at different times depending on how far away they are from the camera. A heterodyne system in which four synchronous samples are taken per beat cycle is analogous to the four-phase stepped homodyne approach, but provides improved performance ${ }^{1 .}$

For a heterodyne system we can determine phase for each pixel using a more general form of the homodyne innerproduct processing from above, provided the image sampling is precisely synchronized with the beat frequency and we acquire an integer number of frames per beat cycle. This algorithm determines the real, $R$, and imaginary, $I$, components of the phase vector using equations (4) and (5) where $N$ is the number of samples per beat cycle, and $A(1: N)$ are the measured values for a given pixel. Equations (6) and (7) are then used to determine phase, $\varphi$, and magnitude, $\alpha$ (signal amplitude), respectively. 


$$
\begin{gathered}
R=\sum_{n=1}^{N} A(n) \sin \left(2 \pi \frac{n}{N}\right) \\
I=\sum_{n=1}^{N} A(n) \cos \left(2 \pi \frac{n}{N}\right) \\
\varphi=\tan ^{-1}\left(\frac{R}{I}\right) \\
\alpha=\sqrt{R^{2}+I^{2}}
\end{gathered}
$$

Although electronically and computationally uncomplicated, homodyne systems using quadrature are known to suffer various limitations, including measurement distortions due to phase contamination from aliasing of harmonics onto the measured signal ${ }^{2}$. Heterodyne systems offer the flexibility to carefully choose the number of samples per cycle such that these distortions can be significantly reduced.

\section{EXTERNAL VS. INTERNAL IMAGE MODULATION}

The fundamental hardware elements required for both homodyne and heterodyne range imagers are the high-speed shutter and light source. Illumination sub-systems use either use LED or laser technology with the choice usually determined by bandwidth and eye-safety requirements. The greatest challenge with these systems is implementing fast global image modulation.

Range imaging detectors can be classified as being either externally or internally modulated. Externally modulated systems employ a separate image modulation device to perform the required shuttering with the resulting image sampled using a standard CCD or CMOS camera. Image intensifiers are often chosen to perform this function because of their ability to provide gain modulation at frequencies up to $100 \mathrm{MHz}$.

Our previous heterodyne system fits in the external modulation category as the shuttering mechanism is provided by an image intensifier. This system is capable of delivering 512 by 512 pixel range images with sub-millimeter depth precision, yet also has the flexibility to operate in faster acquisition modes by compromising spatial resolution and range measurement performance ${ }^{1}$.

Unfortunately the resolution and performance advantages of an externally modulated ranger are offset by a number of disadvantages. Image intensifiers are bulky and costly, suffer from reduced image quality and consume an exorbitant amount of power. Although not an issue for some applications, an example of which would be detailed surface profiling, these factors preclude this type of configuration being used in many other areas such as multimedia integration and mobile robotics.

Internally modulated systems use specialized custom sensors that have both the image modulation and detection functions integrated together on a single monolithic chip. There has been considerable effort by various groups to develop this technology and several devices have been demonstrated ${ }^{6-10}$. Although each approach differs slightly in the way that the photo-generated charge is manipulated, the general principal is consistent for most systems.

Typically a differential structure is employed, using either CCD or CMOS technology, where each pixel contains two modulation gates that are driven out of phase with respect to each other by 180 degrees. Photo-generated charge accumulates in different regions of the pixel (under the modulation gates), depending on the state of the modulation signals, resulting in two samples: one at the initial phase of the shutter signal, the other with the shutter phase shifted by 180 degrees. In the homodyne configuration this means all four samples required to determine $\varphi, \alpha$ and $\beta(A 0, A 180$ and $A 90, A 270$ ) can be obtained from only two images (as opposed to the four required if using an externally shuttered system). 
Currently these sensors are limited in both spatial resolution and depth precision. Array sizes are typically in the order of $25 \mathrm{k}$-pixels and range can only be determined down to sub-centimetre uncertainty levels at best. These drawbacks are due primarily to modulation bandwidth constraints with frequencies generally limited to around $50 \mathrm{MHz}$. Globally modulating an entire array while maintaining clock signal integrity at each pixel is difficult at high frequencies due to factors such as pixel capacitance and clock line impedance.

However, the absence of an external modulation device substantially reduces the power demands, and with the added advantages in cost and size these devices are enabling many new applications. A further advantage is that this configuration uses all available light resulting in a better signal to noise ratio, as opposed to an externally shuttered system that wastes half of the reflected signal.

\section{PMD BASED RANGE IMAGER}

As an alternative to our current intensifier based system, we have developed a range imager using an internally modulated custom sensor - a PhotonICs ${ }^{\circledR}$ PMD $3 k-S$ sensor from PMD Technologies GmbH (Siegen, Germany) ${ }^{11}$. This $64 \times 48$-pixel imager (Fig. 1) is normally used in a homodyne configuration but has the flexibility to also operate in the heterodyne mode. Demodulation at each pixel is achieved using a push-pull differential charge collection and transfer structure (similar to the CCD principle) where photo-generated charge drifts towards one of two wells depending on the relative voltages applied to each photo-gate ${ }^{10}$.

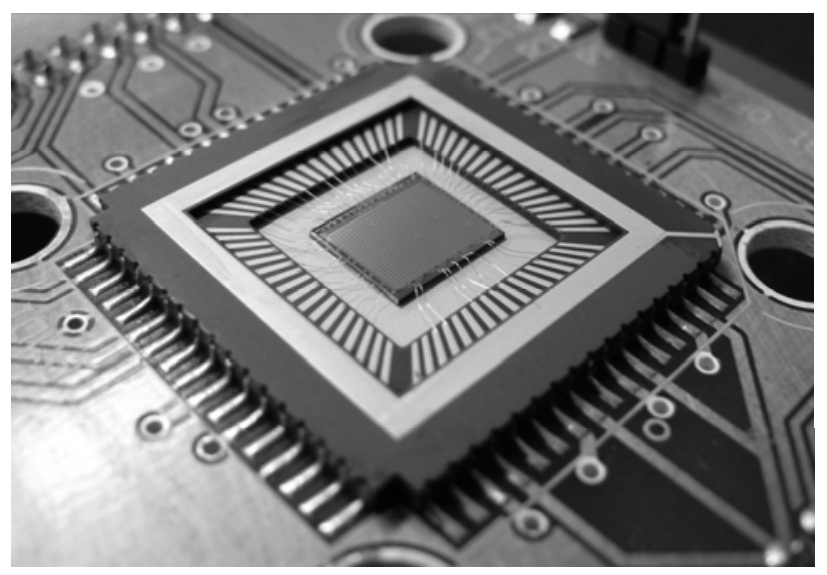

Fig. 1. The PMD 3k-S sensor.

\subsection{Hardware configuration}

Fig. 2 shows a block diagram and a photograph of our PMD based range imager. In addition to the PMD sensor, the system is comprised of several key hardware components. These are illustrated in the block diagram and described in further detail below.

For flexibility in system configuration the PMD sensor is controlled by an XC3S500E Spartan-3E field programmable gate array (FPGA) from Xilinx Inc. (San Jose, CA, USA). This FPGA resides on a development platform that also contains an RS-232 serial link, used as the PC interface for system control and data transfer. Most sensor control and readout signals were interfaced directly to the FPGA, however additional external circuitry was required for driving the pixel modulation clocks and converting the analog pixel outputs to the digital domain.

The cumulative load capacitance due to the high-speed pixel modulation inputs is large, and with no on-chip clock driving capabilities on the PMD sensor this load must be driven by an external source. To ease this burden the sensor is divided into four vertical modulation regions, each with dedicated differential clock pins ( $400 \mathrm{pF}$ per line). In our system these modulation inputs are driven using EL7156 high performance clock drivers (Intersil Corp., Milpitas, CA, USA). These drivers have a high peak drive capability $(3.5 \mathrm{~A})$ allowing modulation of large capacitive loads at up to $40 \mathrm{MHz}$. Another advantage of the driver is that it allows arbitrary setting of the output high and low clock levels, which for the PMD should ideally be $2.4 \mathrm{~V}$ and $0 \mathrm{~V}$ respectively. 
The PMD 3k-S sensor outputs both the raw individual analog pixel voltages (from the two charge collection wells) and a corresponding differential voltage with selectable gain. In our system these values are readout at 3 MSPS (megasamples per second) using an AD9826 3-channel 16-bit imaging signal processor from Analog Devices (Norwood, MA, USA). This device is a flexible high-resolution analog-to-digital conversion stage designed specifically for image sensors.

A Tektronix (Beaverton, OR, USA) AFG3102 dual-channel arbitrary function generator was used to drive the highfrequency (tens of $\mathrm{MHz}$ ) modulation signals for the light source and pixel clocks. With the AFG3102, frequencies can be set with milli-hertz resolution allowing flexibility in beat frequency selection for the heterodyne configuration. It also allows the phase of each channel to be set independently, as required for the homodyne mode. In order to synchronize the sensor and illumination modulation with the imaging system, the function generator was used in burst mode and externally triggered by the FPGA.

Finally, active illumination was provided by a bank of red LED's capable of modulating at frequencies approaching $25 \mathrm{MHz}$. A C-mount lens with adjustable focus and aperture was used to image the reflected modulated light onto the PMD sensor.

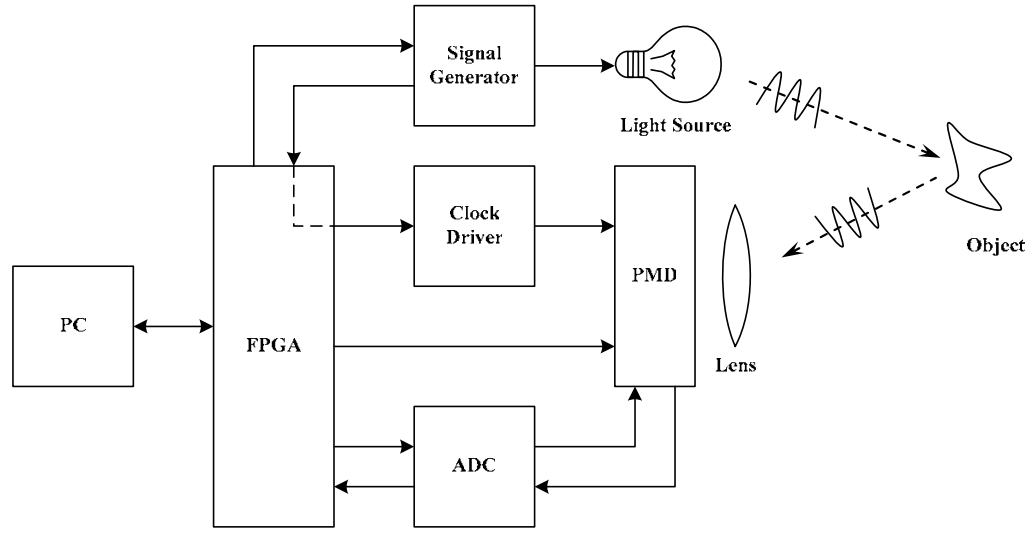

a)

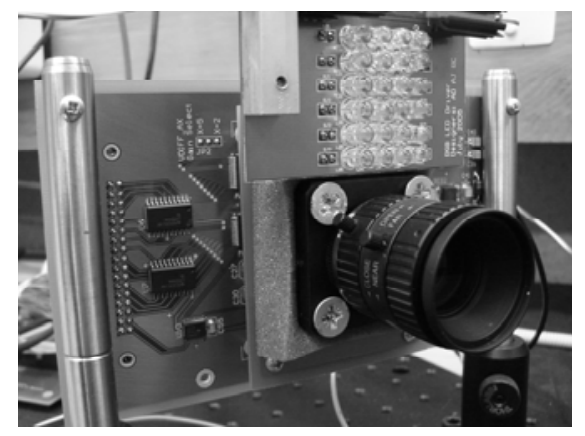

b)

Fig. 2. System diagram, a), and photograph, b).

\subsection{FPGA firmware and system control}

The firmware developed for the FPGA performs two main functions. Firstly it generates all the necessary signals to drive the PMD sensor and read data back from the ADCs; and secondly it provides a control and data-path interface between the system and a PC. With a recommended pixel readout speed of $3 \mathrm{MHz}$, the FPGA system clock was chosen to be $24 \mathrm{MHz}$ (generated using a PLL from a $50 \mathrm{MHz}$ crystal oscillator input).

The frame time for a given image capture is divided into two distinct periods: the integration period when the sensor is modulated and photo-generated charge is collected, followed by the readout period where the captured image is read back to the FPGA. The length of the integration period can be set at a resolution of $1 \mu \mathrm{s}$ up to a maximum of $65.536 \mathrm{~ms}$ allowing flexibility in selecting appropriate frame rates and beat frequencies (for the heterodyne configuration).

During this period an enable signal is asserted to start the function generator outputs (light source and pixel modulation signals). The control of this signal differs depending on the operating. For homodyne, the function generator is only enabled during the integration period, which ensures that both the relative and absolute phases of the two modulation signals are consistent for every frame. For heterodyne the enable signal must be held high for an entire sequence of frames as the modulation signals need to be free-running throughout this period.

In the readout period the sensor control interface clocks analog values out of the sensor row-by-row and presents each pixel output serially to the ADC. The ADC conversion clocks are synchronized with the sensor readout to provide the FPGA with digital data at 3 MSPS, which is then stored inside internal block-RAM. Due to the capacity limitations of this memory, region of interest windowing functionality was added to allow storage of longer image sequences. The total readout time is always constant regardless of the windowing, as the entire array is always clocked out. Therefore total frame time is determined entirely by the chosen length of integration time. 
The other function of the FPGA firmware is to provide a communications interface between the system and a PC. Currently a standard serial RS-232 physical interface is used, which was simple to implement but has limited bandwidth. A simple command protocol was developed in order to issue basic instructions and program various registers or settings from a PC. Each command consists of a single alphabetic character to identify the instruction, followed by any required arguments. Key commands include setting the mode of operation (heterodyne or homodyne), the integration time, number of frames to capture and the region of interest, as well as instructions to start a capture or read measured data. On the PC side, a basic Matlab software interface was written to issue these commands and to appropriately manage data collection. Matlab was also used for all data processing and analysis.

\section{PERFORMANCE CHARACTERIZATION AND MEASUREMENT EXAMPLES}

The primary motivation for building this PMD based system was to implement and characterize our heterodyne configuration using an internally shuttered custom range imaging sensor. Furthermore, it allowed us to compare the measurement performance of both heterodyne and homodyne modes of operation using the same hardware under identical operating conditions.

In order to broadly evaluate key performance characteristics such as linearity and precision (or measurement uncertainty) for the two modes, various cursory experiments were performed. These experiments and their results are outlined below along with various qualitative measurement examples. For all measurements the base modulation frequency and camera frame rate were kept consistent at $22 \mathrm{MHz}$ and 64 frames-per-second respectively. In terms of beat frequency selection for the heterodyne mode, care was taken to ensure an integer number of frames per beat cycle for a given capture. Unless otherwise stated, a sampling rate of five frames per beat was used (for heterodyne), which required a beat frequency of $12.8 \mathrm{~Hz}$. For all quantitative measurements a planar diffuse white surface was used as the target object.

\subsection{Phase linearity}

Ideally range imaging systems will exhibit a perfectly linear relationship between actual and measured phase, however in reality this is not the case. For ease of implementation it is common practice to use square waves to drive the two modulation signals. When mixed together at the camera, the harmonics present in both signals that are above the Nyquist frequency get aliased down to lower frequencies and can contaminate the result ${ }^{2}$.

The phase linearity of our PMD ranger was investigated for both the homodyne and heterodyne (five frames-per-beat) modes of operation. To determine linearity the relative phase between the shutter and the illumination was swept from 0 to 175 degrees in 5 degree increments with range images acquired at each step. Linearity errors were then found by subtracting the actual phase from the measured phase. To help reduce noise and isolate the systematic errors due to harmonic contamination, phase values were averaged spatially over an 8-by-8 pixel region, and across three repeated measurements. The measured linearity errors for the two modes are shown in Fig. 3.

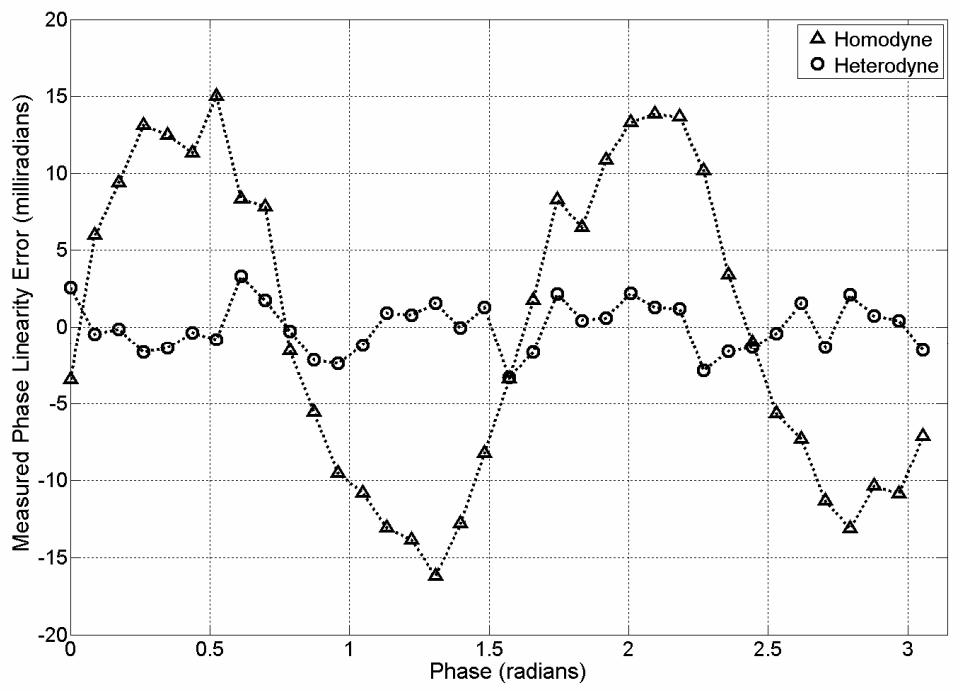

Fig. 3. Linearity error for four-phase quadrature homodyne, and 5 frames-per-beat heterodyne configurations. 
Comparing the two results, linearity errors are significantly worse in the homodyne mode with a root-mean-squared (RMS) error of 10.0 milliradians, as opposed to 1.6 milliradians for heterodyne. For the homodyne case, the harmonic contamination is clearly apparent with the third order harmonic dominating. With four samples per measurement, all odd-valued harmonics are aliased onto the fundamental signal, whereas, in principal the ninth order harmonic is the first to interfere with the beat signal in the five-sample heterodyne case ${ }^{1}$. Due to the triangular shape of the beat signal and the inverse square relationship of its harmonics, the amplitude of the ninth harmonic is significantly attenuated. Also, the inherent rectangular sampling function of the heterodyne mode tends to further attenuate harmonics, as opposed to homodyne where the approach can be considered as sampling with a delta function ${ }^{1}$. The improved linearity of the heterodyne configuration comes at the expense of an extra sample required to construct a range image.

\subsection{Inter- and intra- measurement uncertainty}

Another important characteristic of range imaging systems is their measurement uncertainty. To measure uncertainty for our PMD ranger we captured 100 range images of a flat surface for various modes of operation. Data was acquired for the homodyne configuration, and heterodyne configurations with 4, 5, and 20 frames-per-beat. From this information we could quantify two types of uncertainty for each mode.

Firstly we determined the intra-measurement uncertainty, or the phase variation that can be expected within a given range image. For each range image the standard deviation of the residuals between the measured data and a least-squares planar fit was calculated for a small 8-by-8 pixel region in the centre of the image. Although this ignores any distortions across the image (such as lens and geometric distortions) the region is sufficiently small enough that these nonuniformities have a negligible influence on the results. The mean of all 100 intra-measurement phase (and distance) uncertainty values for each mode are shown in table 1.

Table. 1. Intra-measurement phase and distance uncertainty values for homodyne and heterodyne configurations.

\begin{tabular}{cccc}
\hline Operating Mode & $\begin{array}{c}\text { Number of Samples per } \\
\text { Measurement }\end{array}$ & $\begin{array}{c}\text { 1 } \sigma \text { Phase Uncertainty } \\
\text { (milliradians) }\end{array}$ & $\begin{array}{c}\text { 1 } \sigma \text { Distance Uncertainty } \\
(\mathbf{m m})\end{array}$ \\
\hline Homodyne & 4 & 2.89 & 3.13 \\
Heterodyne & 4 & 2.19 & 2.38 \\
Heterodyne & 5 & 1.78 & 1.93 \\
Heterodyne & 20 & 1.31 & 1.42 \\
\hline
\end{tabular}

The second type of uncertainty considered was the individual pixel phase variation between captures, or the intermeasurement uncertainty. Using the same data-sets as above, the standard deviation of the phase of each pixel across all 100 measurements was calculated (for the same central 8-by-8 pixel region). The mean inter-measurement uncertainty values are reported in table 2 .

Table. 2. Inter-measurement phase and distance uncertainty values for homodyne and heterodyne configurations.

\begin{tabular}{cccc}
\hline Operating Mode & $\begin{array}{c}\text { Number of Samples per } \\
\text { Measurement }\end{array}$ & $\begin{array}{c}\text { 1 } \sigma \text { Phase Uncertainty } \\
\text { (milliradians) }\end{array}$ & $\begin{array}{c}\text { 1 } \sigma \text { Distance Uncertainty } \\
(\mathbf{m m})\end{array}$ \\
\hline Homodyne & 4 & 4.09 & 4.44 \\
Heterodyne & 4 & 3.19 & 3.46 \\
Heterodyne & 5 & 2.56 & 2.78 \\
Heterodyne & 20 & 2.17 & 2.35 \\
\hline
\end{tabular}

Comparing the four-sample homodyne and heterodyne results we see that the intra- and inter- measurement uncertainty values are better for heterodyne by $24 \%$ and $22 \%$ respectively. When using five frames per beat cycle the uncertainty values are better by $38 \%$ and $37 \%$. These results show significant performance gains can be achieved using a heterodyne mode rather than a phase-stepped homodyne configuration. They also show that there is more variation between measurements than there is within a single range image. 


\subsection{Uncertainty versus intensity characteristics}

In order to assess the system's dynamic range performance an experiment was performed to determine phase measurement uncertainty versus active illumination intensity. For each mode (homodyne and heterodyne) a large number of images were captured of a flat surface. Between each acquisition the aperture of the lens was adjusted to vary the amount of light that gets through to the sensor. This had the effect of varying the measured signal amplitude value $(\alpha)$ whilst maintaining consistency in most other factors.

The measured phase and amplitude values for an 8-by-8 pixel region across all captures were pooled into one data set. For a given range of measured intensity values, the standard deviation of their corresponding phase values was calculated. The results for both homodyne and heterodyne are shown in Fig. 4.

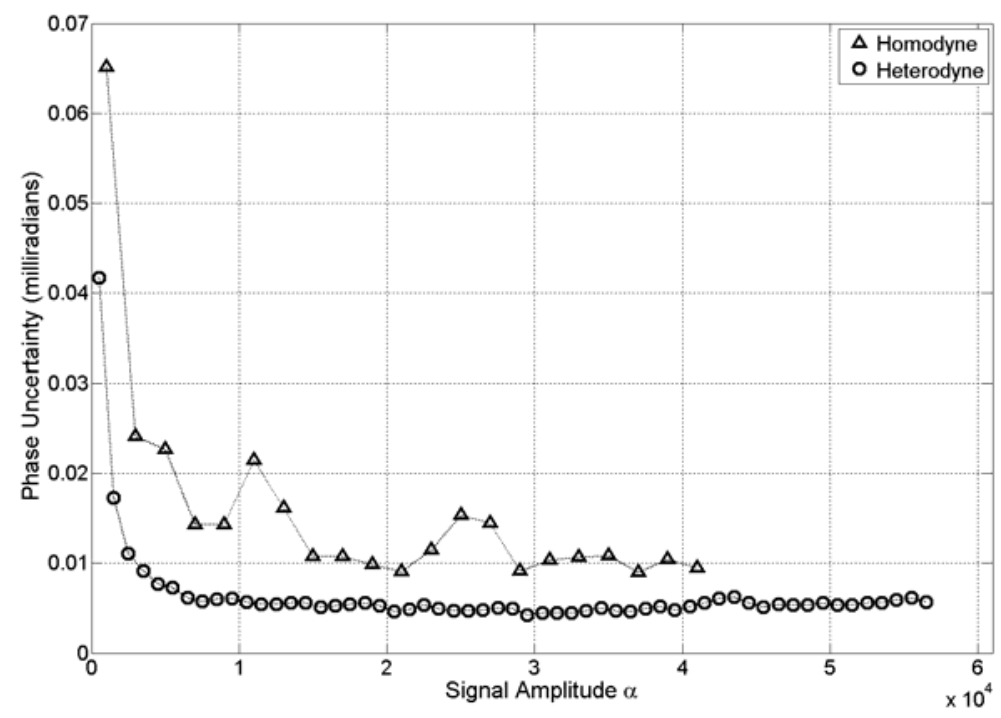

Fig. 4. Intensity versus phase uncertainty for homodyne and heterodyne operating modes.

These results show that the heterodyne mode is able to operate across a large range of intensities whilst maintaining consistent phase uncertainty. The settled uncertainty value here was approximately 4.5 milliradians, which is higher than the values reported for five frame-per-beat heterodyne in section 5.2. This is because pixel-to-pixel phase offsets, due to factors such as the orientation of the flat surface relative to the imaging plane, were not taken into account in this analysis. However these effects were consistent for all measurements as the scene did not change, thus the trend information is valid.

Unfortunately, there was not enough data across all amplitudes to make any valid conclusions about the uncertainty versus intensity performance in the homodyne configuration (as can be seen in Fig. 4). This will investigated further in future work.

\subsection{Measurement examples}

Below are two qualitative measurement examples of objects captured by our system in the heterodyne configuration. Each object was measured over 50 beat cycles (five frames-per-beat) so that the results could be averaged to reduce noise. Fig. 5 shows a single-cycle (upper) and an averaged 50-cycle (lower) range image, as well as a photograph, for each object. In the 3-D surface plots, depth to the camera along the z-axis is indicated by gray level brightness while the $\mathrm{x}$ - and $\mathrm{y}$-axes correspond to the spatial coordinates of the image sensor. The first measurement is of a piece of corrugated foam (Fig. 5. a)) with the range image suggesting the distance between the peaks and troughs of the corrugation is roughly $6 \mathrm{~cm}$ - which in reality it is. The second measurement shows a ceramic bear in the near field up against a flat panel in the background (Fig. 5. b)). Again, the approximate $16 \mathrm{~cm}$ distance between foreground and background was accurately reflected in the measured range image. In both cases the benefit of averaging data over multiple measurements is evident with noticeably smoother surfaces for the 50-cycle averaged results. 

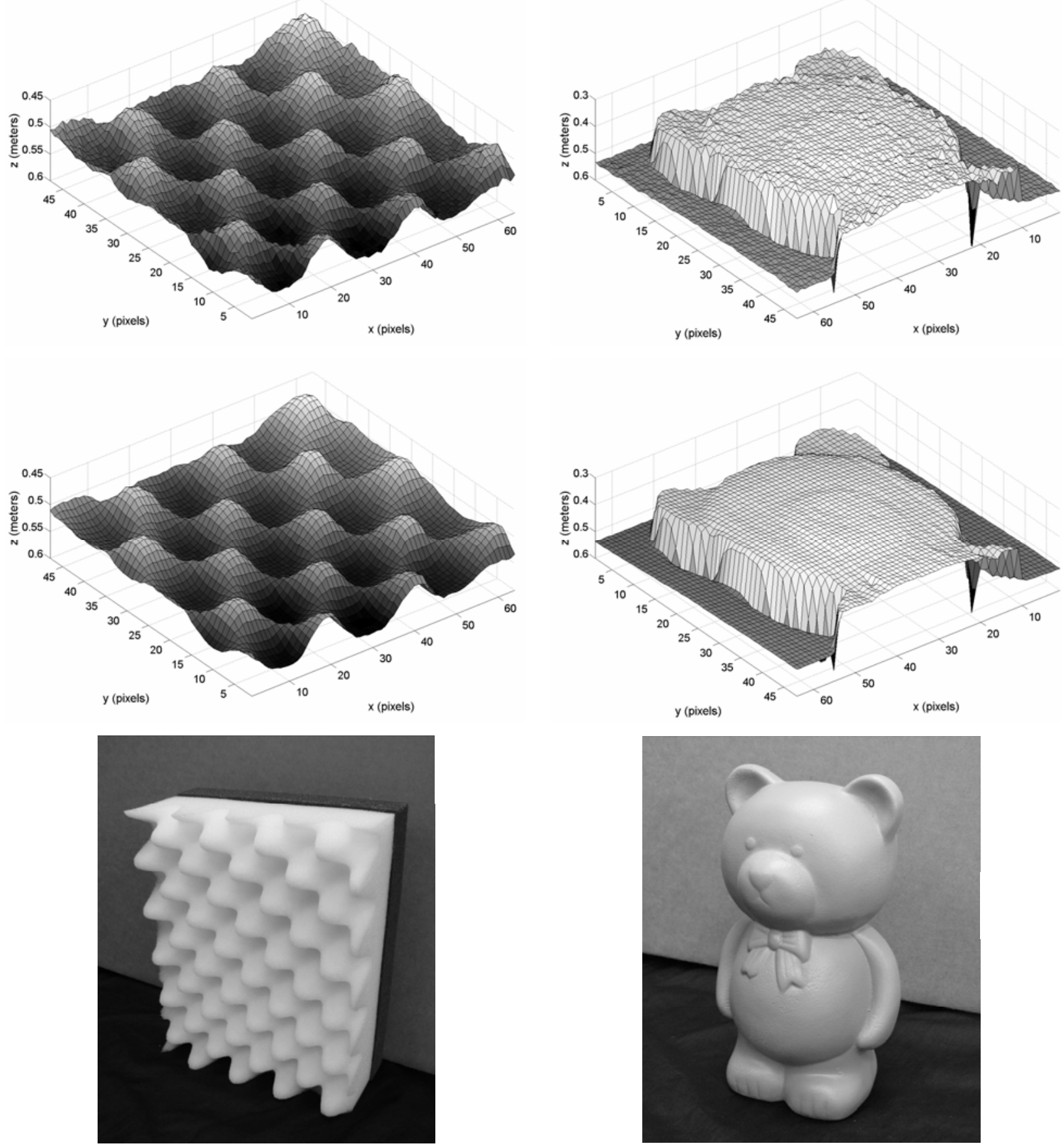

a)

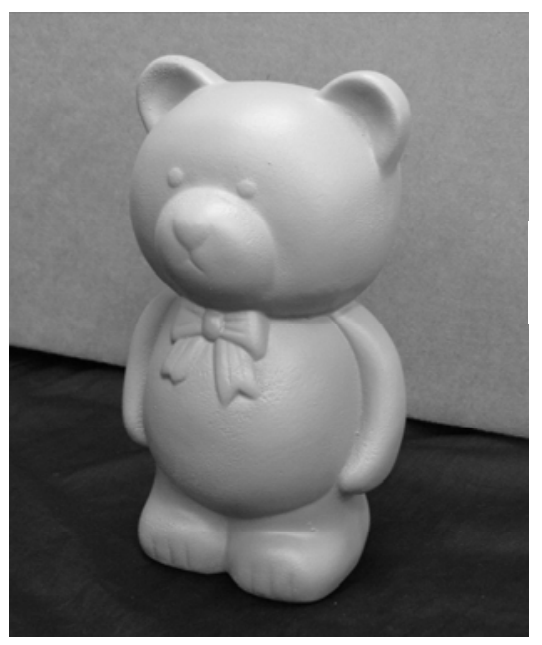

b)

Fig. 5. Single-cycle range image (upper), averaged 50-cycle range image (middle) and photograph (lower) of a) corrugated foam and b) ceramic bear against a flat background. 


\section{CONCLUSION}

The previous generation of our heterodyne range imager used an externally modulated camera configuration that consisted of a bulky and power hungry image intensifier coupled to a standard CCD camera. We have successfully demonstrated a new range imaging system that uses an internally modulated 64-by-48 pixel PMD image sensor. This system has the flexibility to operate in either a heterodyne or a homodyne configuration allowing us to compare the measurement performance of both modes. Results show that better phase linearity can be achieved using a five framesper-beat heterodyne configuration compared to the more common four-sample homodyne mode due mainly to the reduced influence of beat signal harmonics. Also, the heterodyne configuration typically achieves better measurement uncertainty. Although dynamic range analysis was inconclusive for homodyne (due to missing data), the results showed that the heterodyne mode achieves consistent phase uncertainty across a wide range of intensities.

\section{ACKNOWLEDGEMENTS}

The authors would like to thank the Tertiary Education Commission of New Zealand and the Foundation for Research Science and Technology of New Zealand, for financial support.

\section{REFERENCES}

[1] Dorrington, A. A., Cree M. J., Carnegie, D. A., Payne, A. D., Conroy, R. M., Godbaz, J. P. and Jongenelen, A. P. P., "Video-rate or high-precision: A flexible range imaging camera," Proc. SPIE 6813, 681307 (2008).

[2] Payne, A. D., Dorrington, A. A., Cree, M. J. and Carnegie, D. A., "Improved Linearity Using Harmonic Error Rejection in a Full-Field Range Imaging System," Proc. SPIE 6805, 68050D (2008).

[3] Carnegie, D. A., Cree, M. J., Dorrington, A. A., "A High-Resolution Full-Field Imaging System," Review of Scientific Instruments, 76, CID 083702 (2005).

[4] Dorrington, A. A., Cree, M. J., Payne, A. D., Conroy, R. M. and Carnegie, D. A., "Achieving sub-millimetre precision with a solid-state full-field heterodyning range imaging camera," Meas. Sci. Technol., 18, 2809-2816 (2007).

[5] Anthes, J. P., Garcia, P., Pierce, J. T. and Dressendorfer, P. V., "Non-scanned LADAR imaging and applications," Proc. SPIE - Applied Laser Radar Technology, 1936, 11-30 (1993).

[6] Lange, R., Seitz, P., Biber, A. and Lauxtermann, S., "Demodulation Pixels in CCD and CMOS Technologies for Time-of-Flight Ranging," Proc. SPIE, 3965, 177-189 (2000).

[7] Oggier, T., Lehmann, M., Kaufmann, R., Schweizer, M., Richter, M., Metzler, P., Lang, G., Lustenberger, F. and Blanc, N., "An all-solid-state optical range camera for 3D real-time imaging with sub-centimeter depth resolution (SwissRanger)," Proc. SPIE, 5249, 534-545 (2004).

[8] Gokturk, S. B., Yalcin, H., Bamji, C., "A Time-Of-Flight Depth Sensor - System Description, Issues and Solutions," Proc. 2004 IEEE Conf. Computer Vision and Pattern Recognition Workshop, 35 (2004).

[9] Gulden, G., Becker, D., and Vossiek, M., "Novel optical distance sensor based on MSM technology," IEEE Sensors Journal, 4(5), 612-618 (2004).

[10] Xu, Z., Schwarte, R., Heinol, H., Buxbaum, B. and Ringbeck, T., "Smart pixel - photonic mixer device (PMD) new system concept of a 3d-imaging camera-on-a-chip,” PMD Technologies GmbH Tech. rep., (2005), accessed from http://www.pmdtec.com/inhalt/download/documents/paper_4.pdf

[11] PMD Technologies GmbH, http://www.pmdtec.com/e_index.htm 